\title{
Rehabilitation Using Kinect and an Outlook on Its Educational Applications: A Review of the State of the Art
}

\author{
Helena M. Reis ${ }^{1}$, Lucas Wiechmann ${ }^{1}$, Simone S. Borges ${ }^{1}$, Isabela Gasparini' ${ }^{2}$, \\ Monike Tsutsumi $^{1}$, Alexandre F. Brandão ${ }^{3}$, Fernando A. Vasilceac ${ }^{3}$, Adriana \\ Garcia Gonçalves $^{3}$, Ramílio R. R. Filho ${ }^{4}$ e Seiji Isotani ${ }^{1}$ \\ ${ }^{1}$ Universidade de São Paulo (USP) \\ ${ }^{2}$ Universidade do Estado de Santa Catarina (UDESC) \\ ${ }^{3}$ Universidade Federal de São Carlos (UFSCar) \\ ${ }^{4}$ Centro Estadual de Educação Tecnológica Paula Souza (FATEC)
\{helenamcd, sborges, sisotani\}@icmc.usp.br, moniketsutsumi@usp.br, isabela.gasparini@udesc.br, brandaobiotec@gmail.com, fernando.vasilceacloutlook.com, adrigarcia33@yahoo.com.br ramilio.ramalho@outlook.com

\begin{abstract}
Rehabilitation using the Kinect technology and systems for educational purposes can influence patient's motivation. However, approaches that use these two aspects together are poorly investigated. To provide an overview and understanding of the results already obtained in this area, a systematic mapping study was conducted in order to analyze how the rehabilitation using Kinect technology has been investigated. In total, 156 studies were analyzed corresponding to 4 years of research in the area of rehabilitation using Kinect. Among these, 51 studies were related to the development of interfaces and only 50 of them satisfied the inclusion and exclusion criteria defined in this work. As a result, we verified that most of the studies have investigated physical rehabilitation of upper body part. Moreover, the most investigated system type was serious game. We also identified that there are opportunities to research for rehabilitation in educational field.
\end{abstract}

\section{Introduction}

According to the World Report on Disability published by the World Health Organization (WHO) in 2011, more than one billion people worldwide live with some kind of disability. Thus, the international convention on the rights of persons with disabilities highlights the need for rehabilitation, in order to win as much autonomy of physical, mental, social and professional as well as the inclusion and active participation in society. Therefore, services and programs for rehabilitation should be expanded, in addition to continued learning and professional teams (Ministério da Saúde, 2013).

Rehabilitation aims to recover some dysfunction of the human body through physiotherapy exercises (Orlin et al, 2014). This involves the action of performing therapy interventions with or without the assistance of the physiotherapist in order to minimize the cognitive and physical problems of the patient. Nowadays, these 
interventions are being offered with technological support, such as the use of Kinect technology. This technology has been widely used for providing accurate data on the movement of patients in the recovery of locomotors control environment (Chang et al., 2013; Ibarra et al., 2013) or cognitive rehabilitation (González-Ortega et al., 2014). The main advantages of this technology are the portability, ease of use, inexpensive price and, especially, the possibility of system development whose patients could do the exercises in their respectively home. The patient's results would be sent to doctor through a web portal, decreasing the medical cost, infrastructure and human resources. However, those advantages do not always bring motivation for the patient, because he or she would still be under intense and repetitive exercises. It may affect the patient treatment by decreasing their interest in performing the exercises. However, the use of systems for the purpose of entertainment and/or education (e.g. Serious Games) can make patients forget the rehabilitation movements and increase their motivation. Studies on rehabilitation using Kinect for educational purposes have not been widely explored, in spite of its importance. There are few studies in exploiting the gain of rehabilitation along with learning a new concept.

This paper presents the results of a systematic mapping study in order to investigate the rehabilitation with the use of kinect technology form educational purpose. Systematic maps are a way to categorize and synthesize existing information about a particular area of interest, answering impartially of research questions (Petersen 2008). This paper is organized as follows: Section 2 presents the research methodology, the definition of research questions and the conduct of search activities. Section 3 shows the classification of the studies, and Section 4 presents the results of this mapping and the answers of our research questions. In Section 5, it is discussed an outlook on the education using kinect for rehabilitation. Section 6 discusses possible threats to validity, and finally, Section 7 presents the conclusion of the paper.

\section{The systematic mapping process}

This study is based on the guidelines proposed by Petersen et al. (2008) and was conducted according to the following five steps: (i) definition of research questions (RQ), (ii) search for relevant primary studies, (iii) selection of primary studies, (iv) categorization of articles and (v) mapping and extraction of information that answers the research questions previously established. These steps can be applied sequentially and executed several times as required. When all steps are completed, the end result is the systematic mapping.

The research questions must embody the mapping study purpose. Since we aimed at determining how rehabilitation using Kinect has been investigated and the productivity of researchers, our five research questions reflect this purpose as follows:

$\boldsymbol{R} \boldsymbol{Q}_{1}$ : What are the main disabilities types most investigated for rehabilitation systems using Kinect?

$\boldsymbol{R} \boldsymbol{Q}_{1.1}$ : Among the systems for rehabilitation using the Kinect, what is the most investigated disability type with educational purpose?

$\boldsymbol{R} \boldsymbol{Q}_{2}$ : Which body parts the rehabilitation exercises are performed for?

$R_{2}$ : What are the main systems types of the rehabilitation system?

$\boldsymbol{R} \boldsymbol{Q}_{4}$ : What additional devices do the rehabilitation systems usually employ? 
$\boldsymbol{R} Q_{5}$ : What are the main subjects investigated in rehabilitation systems with educational purpose?

Based on these questions we defined the inclusion and exclusion criteria. These criteria are important to identify relevant primary studies that answer the RQs. We determinated the following inclusion criteria:

- If multiple articles show similar studies on the same investigation, only the most recent should be included.

- If there are multiple versions of the same article, e.g. a short article and a complete article, the most complete should be included.

- Articles that describe the development or analysis of the use of Kinect for therapy or rehabilitation for any disability.

And the following exclusion criteria:

- Articles that have no relationship with rehabilitation or therapy using Kinect should be excluded.

- Studies not in English should be excluded.

- Technical reports, documents that are available in the form of short articles or presentations/slides and secondary studies (reviews and systematic mappings of the literature) should be excluded.

The search string used in the academic databases was constructed in two parts, and formatted according to the rules of each database, as follows:

\section{("physical disability" OR "mental disability" OR "physical therapy" OR "physiotherapy rehabilitation" OR "physically handicapped" OR "mental handicapped" OR "motor rehabilitation" OR "accessibility") AND Kinect}

The authors chose to use search strings in English due to the preference for indexed articles in the consolidated databases. The first part of the string is related to disability and therapy, while the second part covers studies related to Kinect technology. No particular field (filter) was used to limit the search, for example, date of publication.

The research of primary studies was performed in the period between $04 / 19 / 2015$ and $04 / 25 / 2015$. The searches were conducted at major electronic databases, all considered likely to return relevant studies in Computer Science, Education and Educational Research (Dybå et al., 2007). Initially, 156 primary papers were retrieved. However, 69 papers were duplicated, resulting 81 candidate papers. After applying the inclusion and exclusion criteria based upon title and abstract, we obtained 51 candidate papers. Finally, after reading introductions and conclusions, we ended up with a final set of 50 primary papers as shown in Table 1 . The complete list of these papers can be obtained at https://goo.gl/iDkqWD.

Table 1 - Number of studies returned by each digital library, total number of candidate studies and final selection.

\begin{tabular}{|l|c|}
\hline \multicolumn{1}{|c|}{ Digital Library } & Quantity \\
\hline ACM Digital Library & 18 \\
\hline Scopus & 67 \\
\hline
\end{tabular}




\begin{tabular}{|l|c|}
\hline Web of Knowledge & 34 \\
\hline Elsevier (via Science Direct) & 3 \\
\hline IEEExplore & 34 \\
\hline \hline Total & $\mathbf{1 5 6}$ \\
\hline Duplicates & $\mathbf{6 9}$ \\
\hline Candidates & $\mathbf{5 1}$ \\
\hline \hline Final Selection & $\mathbf{5 0}$ \\
\hline
\end{tabular}

Figure 1 presents the primary studies clustered by date of publication. There has been a recent growing interest in research on rehabilitation environments using Kinect technology. Although the first publications appeared only in 2011, the number of studies on rehabilitation using Kinect increased six-fold from 2011 to 2014. Importantly, studies with kinect appear only in 2011, due the year that Microsoft released SDK for developing with Kinect. This result reveals that rehabilitation using Kinect technology is becoming a new research trend with room for new findings and improvements.

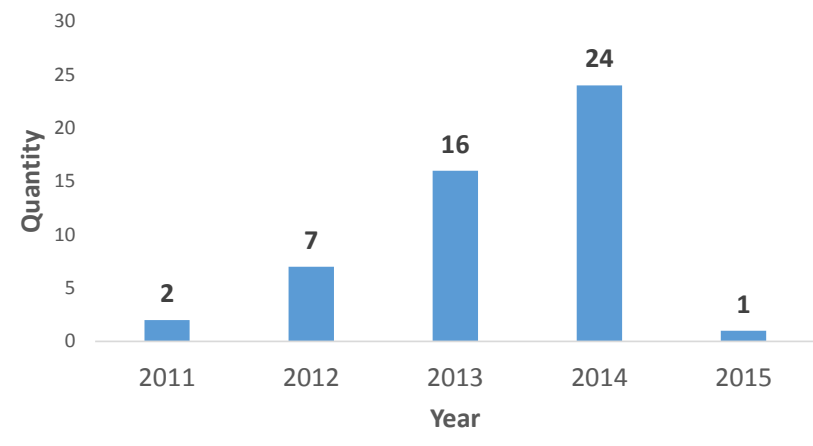

Figure 1: Year-wise distribution of primary studies

\section{Classification according to the study type}

Aiming to answer $\mathrm{RQ}_{1}$ (What are the main disabilities types most investigated for rehabilitation systems using Kinect?), we classified each primary study contribution according to three characteristics: physical, intellectual and visual. These characteristics were based on the Brazilian' law 5.296 ${ }^{1}$, which categorizes the disabilities type in physical, intellectual, visual, multiple and hearing. However, we did not identify any primary studied that could be classified in multiple or hearing category. In Figure 2, we can observe that most primary studies present Kinect technology supports physical disability, within 40 studies (77\%). Thus, we conclude that the answer to $\mathrm{RQ}_{1}$ is that physical disability has been mostly investigated with Kinect support in the rehabilitation. On the other hand, among the selected primary studies, visual disability is

\footnotetext{
${ }^{1}$ http://www.planalto.gov.br/ccivil_03/_ato2004-2006/2004/decreto/d5296.htm
} 
the stage of rehabilitation drawing less attention: only two primary studies, which accounts for only $4 \%$ of the selected studies.

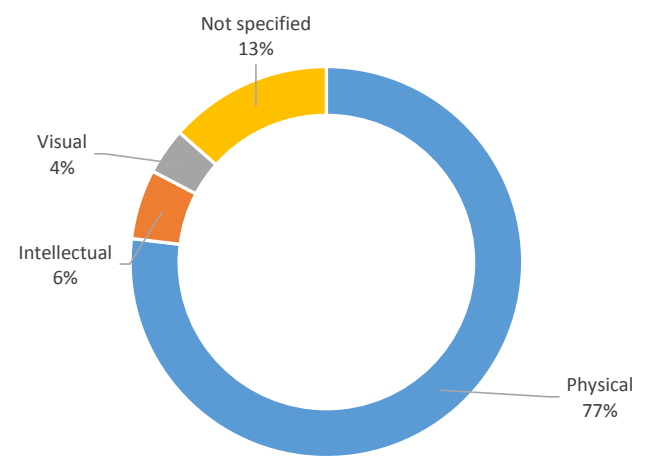

\begin{tabular}{|c|c|c|}
\hline Category & Frequency & $\begin{array}{c}\text { Percentage } \\
(\%)\end{array}$ \\
\hline Physical & 40 & $77 \%$ \\
\hline Not specified & 7 & $13 \%$ \\
\hline Intellectual & 3 & $6 \%$ \\
\hline Visual & 2 & $4 \%$ \\
\hline
\end{tabular}

Figure 2: Categories of disability type

\subsection{Physical}

Our analysis shows that physical rehabilitation research has explored: (i) motor problems in general, which is responsible for 11 primary studies, (ii) wheelchair, explored in 4 studies, (iii) Cerebrovascular accident (CVA) with 10 studies, (iv) multiple sclerosis with 4 studies, (v) cerebral palsy with 7 studies, (vi) facial palsy, (vii) shoulder impingement syndrome and (viii) post-polio syndrome make up 1 study each.

From the Studies found for rehabilitation of stroke patients, eight of ten used Serious Game to support the rehabilitation. Serious Game is also extensively explored for the rehabilitation of people with multiple sclerosis, cerebral palsy, facial and impingement syndrome.

\subsection{Intellectual}

In this category we included all studied that proposed the use of Kinect in the rehabilitation for intellectual disabilities. The main disabilities investigated in this category were autism spectrum disorder and dementia. Ranatunga et al. (2012) explored the use of Kinect together with humanoid/NAO to assist in the rehabilitation of children with autism spectrum disorder. The therapist used the humanoid to teach body language, such as movement of hands and arms, in order to motivate the patient to participate and improve their motor skills.

Another study investigating the use of humanoid to motivate the rehabilitation exercises performing was proposed by Guneysu et al. (2014). In this study, the authors proposed that children should mimic the movements of the humanoid for the rehabilitation of the upper body.

Most of the studies selected in this category exploit rehabilitation for children who have some dementia or autistic spectrum disorder (Ranatunga et al., 2012; Williams, 2013; Guneysu et al., 2014; González-Ortega et al., 2014). Of these, only two (Williams, 2013) have the system facing serious games and three (Ranatunga et al., 2012; Williams et al., 2013; Guneysu et al., 2014) are targeted to children. 


\subsection{Visual}

The primary studies, which were classified into this category, investigated the use of Kinect for blind and low vision to exercise. Rector et al. (2013) proposed a system in which patients could participate in yoga classes. Once patients trigger the voice commands, the Kinect camera verifies the yoga posture and, if necessary, the system can give new instructions by voice to the patient, in order to improve or correct their posture. The target audience of this system was adults and was rated by 16 blind and low vision patients. When we remove the technology from the patient, they might find the exercise more difficult, which will decrease your physical activity.

Although the study by Brock and Kristensson (2013) is focused on visually impaired, rehabilitation is not addressed. In this study, the authors have investigated the use of Kinect to guide patients in different environments. The Kinect recognizes the obstacles and drives the echolocation to make sounds to the patient and inform him to divert barrier.

\section{Analysis}

In this section, we analyze the results of our mapping study. The purpose of this section is to give an overview of how Kinect has been used to support the rehabilitation. The information drawn from the selected primary studies is also used to answer our mapping study's research questions.

Figure 3 shows a conceptual map on using Kinect physical and intellectual rehabilitation. They were separated into four distinct groups: system type, additional device, disability type, and body part. (i) System type: the kind of system in which the patient is interacting (e.g. serious games or physiotherapeutic exercises); (ii) Additional device: device besides Kinect used to assist the patient ; (iii) Disability type: the kind of disability that patient has and (iv)Body part: which part of body is studied..

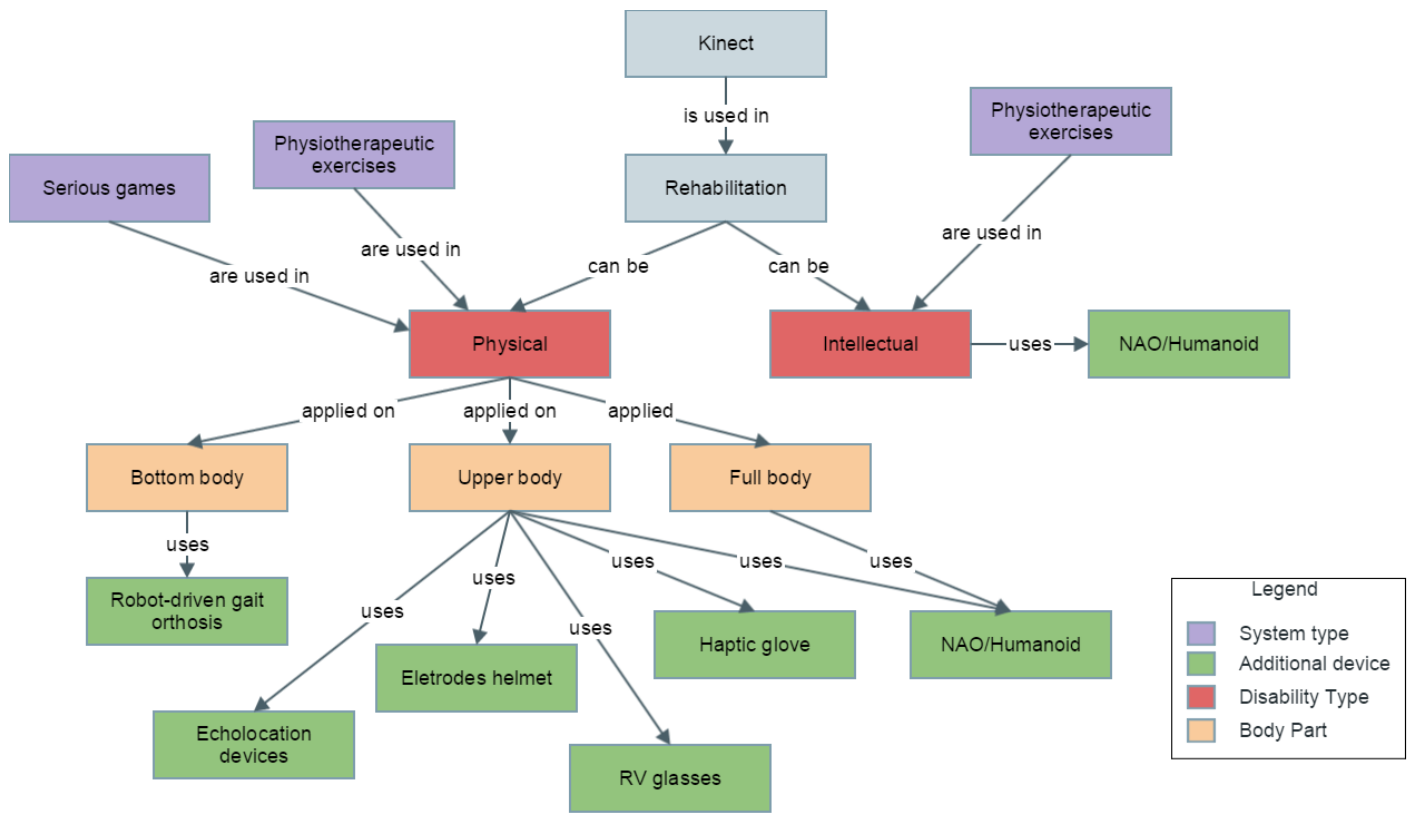

Figure 3: Concept map used for the classification of the selected papers 
From Table 3, it can be seen that most of the selected primary studies investigate upper body part rehabilitation (50\%), which provided an answer to $\mathrm{RQ}_{2}$ (Which body parts the rehabilitation exercises are performed for?). We considered upper part as head, face, shoulders, arms and hands. In the other hand, the lower part is the less investigated, with only 5 primary studies $(10 \%)$. A possible explanation, for the lack of studies on the lower body, is the need to use additional devices that assist in rehabilitation, since these devices are expensive and difficult to maintain.

Table 3 - Body parts investigated on primary studies

\begin{tabular}{|c|c|c|}
\hline Body part & Frequency & Percentage (\%) \\
\hline Upper & 25 & $50 \%$ \\
\hline Full body & 18 & $36 \%$ \\
\hline Lower & 5 & $10 \%$ \\
\hline Not specified & 2 & $4 \%$ \\
\hline
\end{tabular}

Through table 4, we can also identify what is the main system type in rehabilitation, which answers $\mathrm{RQ}_{3}$ (What are the main systems type of the rehabilitation system?). Serious Game, with $64 \%$ of all primary studies, is the most investigated system type. Serious games are usually used to increase the motivation of patients to perform the rehabilitation movements. Patients tend to be more attentive to carry out game tasks prone to make them forget the pain caused by the rehabilitation movements.. The primary studies classified as "Others" refers to studies that have no system for interaction. As an example, the authors of the study Brock and Kristensson (2013) use Kinect for the visually handicapped to locate themselves in the environment. Another example is the study of Gerling et al. (2013) in which the patient uses the Kinect for getting the wheelchair through gestures made by the head.

Table 4 - Primary studies categorized according to system type

\begin{tabular}{|c|c|c|}
\hline Type & Frequency & Percentage (\%) \\
\hline Serious games & 32 & $64 \%$ \\
\hline Physiotherapeutic exercises & 16 & $32 \%$ \\
\hline Others & 2 & $4 \%$ \\
\hline
\end{tabular}

Most primary studies do not use devices other than Kinect to aid in rehabilitation, representing 39 primary studies (79\%), which provided an answer to RQ 4 (What additional devices the rehabilitation systems usually use?). On the other hand, the most frequently used additional device is NAO/Humanoid with 4 primary studies $(8 \%)$. The NAO/Humanoid was used for rehabilitation with autistic children and elderly people with motor problems, in order to increase motivation along the rehabilitation process. Table 5 shows the types of additional devices, which were classified into seven categories.

Table 5 - Primary studies categorized according to additional devices

\begin{tabular}{|c|c|c|}
\hline Additional device & Frequency & Percentage (\%) \\
\hline None & 39 & $76 \%$ \\
\hline NAO/Humanoid & 4 & $8 \%$ \\
\hline Robot-driven gait orthosis & 3 & $6 \%$ \\
\hline
\end{tabular}




\begin{tabular}{|c|c|c|}
\hline Haptic glove & 2 & $4 \%$ \\
\hline Virtual Reality glasses & 1 & $2 \%$ \\
\hline Echolocation devices & 1 & $2 \%$ \\
\hline Eletrodes helmet & 1 & $2 \%$ \\
\hline
\end{tabular}

Despite the large number of primary studies do not describe the age group rehabilitation systems have been developed (37\%), at least, 20 primary studies presented rehabilitation systems aimed for adults, and that represents $38 \%$ of the studies. However, few studies investigate the rehabilitation using Kinect for the elderly $(8 \%)$ and adolescents $(6 \%)$. Table 6 shows the age groups in which the rehabilitation systems have been developed.

Table 6 - Primary studies categorized according to age group

\begin{tabular}{|c|c|c|}
\hline Type & Frequency & Percentage (\%) \\
\hline Adult & 20 & $38 \%$ \\
\hline Not specified & 19 & $37 \%$ \\
\hline Child & 6 & $11 \%$ \\
\hline Elderly & 4 & $8 \%$ \\
\hline Adolescent & 3 & $6 \%$ \\
\hline
\end{tabular}

\section{An outlook on the education using Kinect for rehabilitation}

We believe that rehabilitation with Kinect using educational systems can promote benefits to the patient in two ways: first, improving their cognitive and physical capacity; and second, aiding them acquiring the new knowledge. Together, this approach and serious games have the potential to motivate patients and to diminish their discomfort and pain, when performing rehabilitation exercises,

Among primary studies returned investigating the use of Kinect to assist in the rehabilitation of patients, only two studies have educational purposes (Table 7). One is focused on physical rehabilitation (blind) and one for mental rehabilitation (autism spectrum disorder), which provides the answer to $\mathrm{RQ}_{1.1}$ (Among the systems for rehabilitation using the Kinect, what are the main disability type most investigated with educational purpose?).

Aiming to answer $\mathrm{RQ}_{5}$ (What are the main subjects investigated in rehabilitation systems with educational purpose?), we have identified the subjects investigated by the authors of the primary studies. Rector et al. (2013) proposes the use of Kinect for the purpose of yoga teaching positions for blind adult. In this system, the patient should perform physiotherapeutic exercises. Also, Ranatunga et al. (2012) investigates the use of humanoid/NAO to teach children with intellectual (autism spectrum disorder) problems to understand the parts of the human body. The authors used serious game in this study to motivate children to perform the exercises. Both studies found for educational purposes are directed learning extracurricular subjects. This means there is a lack of rehabilitation systems in order to teach traditional subjects (e.g. mathematics, geography, history) 
Table 7 - Primary studies categorized according to system type

\begin{tabular}{|c|c|c|c|}
\hline Desability type & Age group & System type & Subject \\
\hline Physical (blind) & Adult & Physiotherapy exercises & Yoga \\
\hline Mental (autism spectrum disorder) & Child & Serious game & Human body \\
\hline
\end{tabular}

Our results indicate that there has not been so much interest in rehabilitation using Kinect for educational proposes. Although the investigation in this field is recent, only two primary studies were found in four years of research.

\section{Threats to validity}

To ensure an unbiased selection, the research questions and the inclusion and exclusion criteria were defined at the beginning of the mapping. However, a threat related to assessing the quality of the included studies cannot be ruled out, as the studies were selected without assigning scores. Another possible threat identified is the possibility that some relevant articles were not included due to the use of a limited collection of digital libraries. The classification system and elaborate categories also represent a threat to validity. As shown by Pretorius and Budgen (2008), the best way to sort and categorize results is only obtained at the end of the selection. Furthermore, the grouping of studies in more than one category can represent a potential threat to assessing frequency and considering statistics in this mapping study.

\section{Concluding remarks}

The main purpose of our mapping study is to provide an overview of what has been investigated in the context of Kinect applied to rehabilitation. To fulfill our goal, we followed a systematic methodology, i.e., systematic mapping. We defined six research questions to be answered by our mapping. $R Q_{1}$ : What is the main disability types most investigated for rehabilitation systems using Kinect? RQ $Q_{1.1}$ : Among the systems for rehabilitation using the Kinect, what are the main disability types most investigated with educational purpose? $R Q_{2}$ : Which body parts the rehabilitation exercises are performed for? $R Q_{3}$ : What are the main systems types of the rehabilitation system? $R Q_{4}$ : What additional devices the rehabilitation systems usually use? $R Q_{6}$ : What are the main subjects investigated in rehabilitation systems with educational purpose? According to our results, most studies investigated physical rehabilitation $\left(R^{2} Q_{1}\right.$ see Figure 2$)$ to upper body part $\left(\mathrm{RQ}_{2}\right.$ - see Table 3$)$ with none additional device $\left(\mathrm{RQ}_{3}-\right.$ see Table 4). We also identified that serious games are the most investigated system type for rehabilitation using Kinect $\left(\mathrm{RQ}_{4}-\right.$ see Table 5). In the educational context, few studies have proposed the use of Kinect for rehabilitation with educational purpose, within only two studies, and one is for physical and intellectual rehabilitation $\left(\mathrm{RQ}_{1.1}-\right.$ see Table 7). The subjects investigated in these studies were: teaching of yoga for blind people and teaching human body for autism spectrum disorder $\left(\mathrm{RQ}_{5}-\right.$ see Table 7$)$.

This research brings a novelty, which is the first systematic mapping covering research into rehabilitation using Kinect. Another contribution of this research is the results about what system type, body part, and additional devices have been most investigated in rehabilitation using Kinect. By analyzing such table, it is possible to identify that there are few studies about rehabilitation using Kinect with educational 
purpose, which can bring us a conclusion: this is an important field and an opportunity of research.

\section{Acknowledgments}

The authors want to thank CNPq and CAPES for supporting this research.

\section{References}

Brock, M., \& Kristensson, P. O. (2013). Supporting Blind Navigation Using Depth Sensing and Sonification. In Proceedings of the 2013 ACM Conference on Pervasive and Ubiquitous Computing Adjunct Publication (pp. 255-258). New York, NY, USA: ACM.

Chang, Y.J., Han, W.Y., Tsai, Y.C.(2013). A Kinect-based upper limb rehabilitation system to assist people with cerebral palsy. Res. Dev. Disabil. 34 (11), 3654-3659.

Dybå, T. Dingsøyr, T. and Hanssen, G. K. (2007). Applying Systematic Reviews to Diverse Study Types: An Experience Report. In 1st International Symposium on Empirical Software Engineering and Measurement, pages 225-234.

González-Ortega, D., Díaz-Pernas, F. J., Martínez-Zarzuela, M., \& Antón-Rodríguez, M. (2014). A Kinect-based system for cognitive rehabilitation exercises monitoring. Computer Methods and Programs in Biomedicine, 113(2), 620-631.

Guneysu, A., Siyli, R. D., \& Salah, A. A. (2014). Auto-evaluation of motion imitation in a child-robot imitation game for upper arm rehabilitation. In Robot and Human Interactive Communication, 199-204.

Ibarra, J.M.Z., Tamayo, A.J., Sánchez, A.D., Delgado, J.E., Cheu, L.E., Arévalo, W.A.(2013). Development of a system based on $3 D$ vision, interactive virtual environments, ergonometric signals and a humanoid for stroke rehabilitation. Computer Methods and Programs in Biomedicine, 112 (2), 239-249

Kitchenham, B. Charters, S.(2007) Guidelines for performing systematic literature reviews in software engineering. Technical report, Keele University and University of Durham.

Orlin, M.N., Cicirello, N.A., O'Donnell, A.E., Doty, A.K. (2014). The Continuum of Care for Individuals With Lifelong Disabilities: Role of the Physical Therapist. Physical Therapy, 94(7), 1043-1053

Petersen, K., Feldt, R., Mujtaba, S. \& Mattsson, M. (2008) Systematic mapping studies in software engineering. Proceedings of the 12th international conference on Evaluation and Assessment in Software Engineering, 68-77.

Pretorius, R. e Budgen, D. (2008) A Mapping Study on Empirical Evidence Related to the Models and Forms used in the UML. In 2nd ACM-IEEE International Symposium on Empirical Software Engineering and Measurement (ESEM), pages 342- 344.

Ranatunga, I., Torres, N. A., Patterson, R., Bugnariu, N., Stevenson, M., \& Popa, D. O. (2012). RoDiCA: A Human-robot Interaction System for Treatment of Childhood Autism Spectrum Disorders. In Proceedings of the 5th International Conference on PErvasive Technologies Related to Assistive Environments 50:1-6.

Rector, K., Bennett, C. L., \& Kientz, J. A. (2013). Eyes-free Yoga: An Exergame Using Depth Cameras for Blind and Low Vision Exercise. In Proceedings of the 15th International ACM SIGACCESS Conference on Computers and Accessibility, 12:1-8.

Williams, C. (2013). Evaluating Therapeutic Engagement and Expressive Communication in Immersive Multimedia Environments. In Proceedings of the 7th International Conference on Universal Access in Human-Computer Interaction, 514-523. 